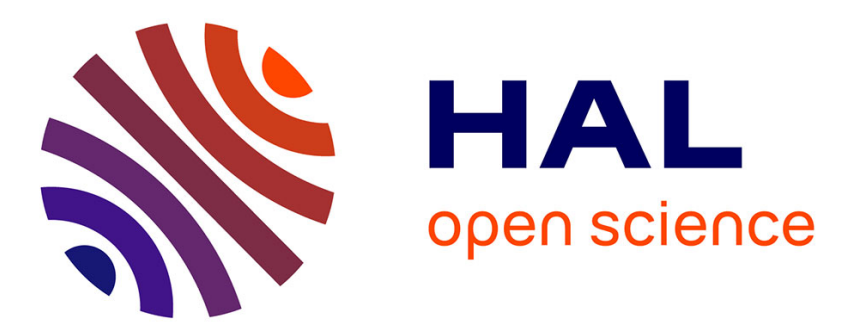

\title{
Mise en évidence et quantification des couplages thermomécaniques réversibles et irréversibles dans les bétons sains et endommagés par des cycles gel-dégel
}

\author{
Vincent Huon, Bruno Cousin, Olivier Maisonneuve
}

\section{- To cite this version:}

Vincent Huon, Bruno Cousin, Olivier Maisonneuve. Mise en évidence et quantification des couplages thermomécaniques réversibles et irréversibles dans les bétons sains et endommagés par des cycles gel-dégel. Comptes rendus de l'Académie des sciences. Série IIb, Mécanique, 2001, 319, pp.331-335. 10.1016/S1620-7742(01)01341-1 . hal-02457400

\author{
HAL Id: hal-02457400 \\ https://hal.science/hal-02457400
}

Submitted on 28 Jan 2020

HAL is a multi-disciplinary open access archive for the deposit and dissemination of scientific research documents, whether they are published or not. The documents may come from teaching and research institutions in France or abroad, or from public or private research centers.
L'archive ouverte pluridisciplinaire HAL, est destinée au dépôt et à la diffusion de documents scientifiques de niveau recherche, publiés ou non, émanant des établissements d'enseignement et de recherche français ou étrangers, des laboratoires publics ou privés. 


\title{
Mise en évidence et quantification des couplages thermomécaniques réversibles et irréversibles dans les bétons sains et endommagés par des cycles
} gel-dégel

\author{
Vincent HUON, Bruno COUSIN, Olivier MAISONNEUVE \\ Laboratoire de mécanique et génie civil, UMR 5508, CNRS-UMII, Université Montpellier II, \\ place Eugène Bataillon, 34095 Montpellier cedex 05, France \\ Courriel : huon@Imgc.univ-montp2.fr; cousin@Imgc.univ-montp2.fr; maisonneuve@Imgc.univ-montp2.fr
}

Résumé. À partir de l'observation fine par thermographie infrarouge des phénomènes thermiques mis en jeu lors de sollicitations de compression quasi-statiques, sont identifiées et quantifiées l'incidence de l'effet thermoélastique et celle de la dissipation intrinsèque associée à l'état d'endommagement d'un béton ayant subi des cycles gel-dégel.

rhéologie / bétons / endommagement / couplages thermomécaniques / thermographie infrarouge

\section{Showing and measuring of reversible or not thermomechanical couplings in undamaged and freezing/thawing damaged concrete}

\begin{abstract}
We study thermal phenomena which occur during compressive loading tests on some
Abstract.
damaged or not concrete. Two calorific effects are shown through an experimental study using infrared thermography. The first, intrinsically reversible, is due to the thermoelastic coupling. The second is due to damage concrete state.
\end{abstract}

rheology / concrete / damage / thermomechanical couplings / infrared thermography

\section{Introduction}

En raison de l'importance des bétons dans le domaine du génie civil, de très nombreux travaux ont porté sur leur comportement mécanique, sous diverses conditions d'environnement et de charge. Partant d'un comportement macroscopique élastique, les auteurs se sont attachés à explorer expérimentalement et à modéliser l'endommagement des bétons et une de ses conséquences : le comportement unilatéral [1-5].

Considérant les bétons comme des matériaux élastiques endommageables, le travail présenté concerne la mise en évidence et la quantification, lors d'essais de compression quasi-statique, des effets thermiques associés au comportement élastique (couplage thermoélastique) et à l'endommagement (phénomène dissipatif) d'un béton ayant subi des cycles gel-dégel. 


\section{Dispositif expérimental et type d'essais}

Le dispositif comporte une machine d'essais mécaniques couplée à une caméra infrarouge. Il est possible de suivre en parallèle l'évolution des paramètres mécaniques tels que la force et l'allongement et celle de la carte de température de la face observée de l'éprouvette. Les sollicitations sont des cycles charge-décharge quasi-statiques, en compression, à vitesse de déplacement de traverse imposée.

La caractérisation thermomécanique de l'évolution de l'endommagement d'un béton pour des sollicitations dynamiques $(100 \mathrm{~Hz})$ a été faite par vibrothermographie (Luong [6]). Pour des sollicitations quasistatiques, sur des éprouvettes à endommagement constant, les faibles puissances mises en jeu et l'importance de la chaleur spécifique conduisent, dans les conditions de l'expérience, à l'observation de très petites variations de température. Ceci constitue une difficulté majeure.

\section{Matériau étudié}

Les essais ont été mis en œuvre principalement sur des éprouvettes parallélépipédiques $(50 \times 150 \times 300)$ en microbéton composé de sable siliceux $(0$ à $4 \mathrm{~mm})$ avec des rapports eau/ciment $(E / C)$ et ciment/sable $(C / S)$ respectivement de 0,5 et 0,3 .

La résistance en compression à 28 jours est d'environ $40 \mathrm{MPa}$. Ces éprouvettes ont été endommagées au cours de cycles gel-dégel par le Norvegian Building Research Institute [7]. Elles ont subi 14 cycles rapides gel-dégel avec une variation de température d'environ $12^{\circ} \mathrm{C}$ par heure entre les limites $5^{\circ} \mathrm{C}$ et $-18^{\circ} \mathrm{C}$.

L'endommagement obtenu est invisible à l'œil nu. Cependant une analyse sous microscope [7], d'une section de $50 \times 150 \mathrm{~mm}^{2}$, a révélé un réseau de fissuration dense avec une distance moyenne entre fissure de 0,5 à $1 \mathrm{~mm}$ et une largeur de fissure de l'ordre de 10 à $20 \mu \mathrm{m}$, pour la plupart localisées dans l'auréole de transition.

Lors de la sollicitation mécanique, l'évolution de la température de l'éprouvette résulte a priori de deux phénomènes concomitants : l'un de nature intrinsèquement réversible (dissipation intrinsèque nulle), l'autre non. Le souhait étant de les identifier séparément, il convient d'en caractériser isolément un au moins, l'effet thermoélastique, dans des conditions expérimentales permettant de négliger les couplages entre les deux phénomènes en raison de leur faiblesse relative.

\section{Mise en évidence de l'effet thermoélastique}

Ne disposant pas d'éprouvettes «saines » correspondant au béton précédemment décrit, il a été procédé à des essais cyclés de compression sur des éprouvettes «non endommagées» en béton ordinaire de composition à peu près similaire $(E / C=0,5 ; C / S=0,3)$ et en béton de poudres réactives $(\mathrm{BPR})$ (Richard et al. [8]).

Pour le premier matériau, il a été observée une variation de température en phase avec le chargement, positive, avec retour à zéro en fin de décharge et d'amplitude maximale de $0,07^{\circ} \mathrm{C}$ après filtrage. La contrainte maximale atteinte au cours de ces essais était de $30 \mathrm{MPa}$. Les écarts de température constatés sont faibles par rapport au $0,2^{\circ} \mathrm{C}$ correspondant au bruit de la caméra. Cela a conduit au souhait de mettre en évidence le même effet sur un BPR, supportant des contraintes plus importantes, et de comparer les résultats expérimentaux à une estimation théorique.

Trois cycles charge-décharge ont été réalisés sur les éprouvettes en BPR, jusqu'à une contrainte de l'ordre de $160 \mathrm{MPa}$ avec une vitesse de traverse de $5 \times 10^{-2} \mathrm{~mm} \cdot \mathrm{s}^{-1}$ identique pour les trois cycles. La figure 1 fait correspondre à chaque état du chargement mécanique (courbe blanche), les variations de température $\left(\theta=T-T_{0}\right)$, au cours du temps, le long de l'axe médian de la face observée de l'éprouvette.

On constate à nouveau une variation positive de la température, constante le long de l'éprouvette aux effets de bord près, en phase avec l'évolution de la charge qu'il est logique d'associer à l'effet thermoélastique. 


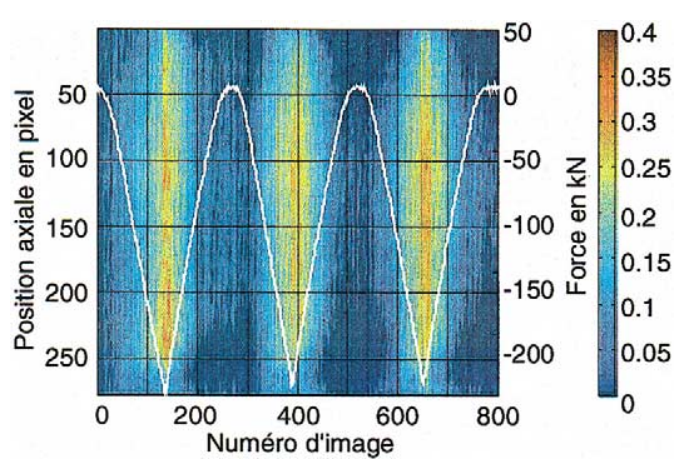

Figure 1. Evolution au cours du temps du profil de température de l'axe médian de l'éprouvette (en abscisse $1 \mathrm{~s}=6,25$ images et en ordonnée de gauche 270 pixel $=80 \mathrm{~mm}$ ). Courbe blanche en surimpression : évolution de la charge.

Figure 1. Time evolution of the length-wise temperature profile $(1 \mathrm{~s}=6.25$ images and 270 pixel $=80 \mathrm{~mm})$. Surimposed white curve: load evolution.

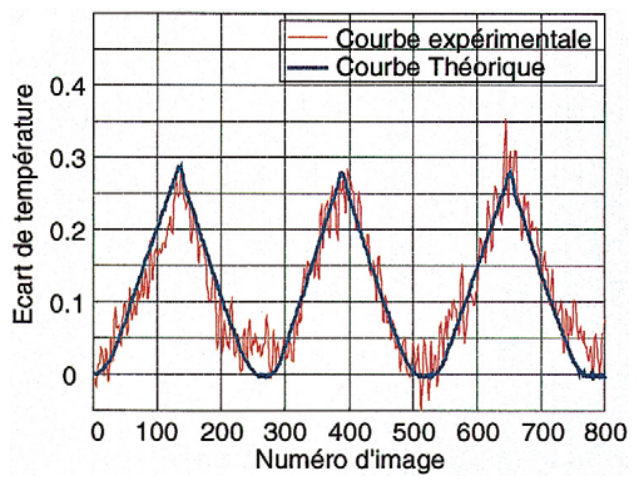

Figure 2. Evolution au cours du temps de la variation de température au point milieu de l'éprouvette. Comparaison courbe expérimentale et théorique.

Figure 2. Time evolution of temperature variation at middle sample point. Experimental and theoretical curve comparison.

\section{Estimation de l'effet thermoélastique}

On part pour cela de l'équation linéarisée de la chaleur, avec comme seul terme source au second membre celui correspondant au couplage thermoélastique, pour une sollicitation de compression uniaxiale, le matériau étant supposé thermoélastique linéaire homogène isotrope. La linéarisation est justifiée par la faiblesse des phénomènes thermiques mis en jeu. Les observations expérimentales (variations de température en phase avec la charge) et les caractéristiques thermiques des matériaux considérés (faible diffusivité et forte chaleur spécifique) permettent de faire l'hypothèse de quasi-adiabaticité locale durant l'essai. L'équation de la chaleur peut alors s'écrire, après les simplifications correspondantes :

$$
\rho C \frac{\partial \theta}{\partial t}=-\left(\theta+T_{0}\right) E \alpha \dot{\varepsilon}
$$

où $\rho, C, \alpha, E$ et $\dot{\varepsilon}$ sont respectivement, la masse volumique, la capacité thermique massique, le coefficient de dilatation, le module d'Young et la vitesse de déformation.

En intégrant (1) avec la condition initiale $\theta=0$ à $t=0$, on obtient, $\theta$ étant toujours très petit devant $T_{0}$ :

$$
\theta=-\frac{T_{0} \alpha \sigma}{\rho C}
$$

où $\sigma$ est la contrainte dans le matériau à l'instant considéré.

On peut alors comparer (figure 2) l'évolution de $\theta$ observée expérimentalement, lorsque $T_{0}=298 \mathrm{~K}$ et $\rho=2,5 \times 10^{3} \mathrm{~kg} \cdot \mathrm{m}^{-3}$, avec celle obtenue par (2) en prenant : $C=838 \mathrm{~J} \cdot \mathrm{kg}^{-1} \cdot \mathrm{K}^{-1}$ (Lemaitre et Chaboche [9]) et $\alpha=12 \times 10^{-6} \mathrm{~K}^{-1}$, valeur de référence donnée pour les bétons à granulats entièrement siliceux (BPEL 91).

On note une remarquable adéquation entre l'expérience et les prévisions du modèle. Il est légitime de conclure à une mise en évidence et quantification correctes de l'effet thermoélastique sur un BPR. Remarquons que, si l'on adopte pour les bétons ordinaires testés la même valeur que précédemment pour la capacité thermique massique et le coefficient de dilatation (la nature des constituants n'étant pas 
fondamentalement différente de celle d'un BPR), on calcule, pour une contrainte maximale de compression de $30 \mathrm{MPa}$, une variation de température maximale de $0,07^{\circ} \mathrm{C}$ identique à celle observée dans des conditions limites de sensibilité de mesure.

\section{Identification des effets thermiques associés à un état d'endommagement}

Les essais ont été conduits sur les éprouvettes décrites au paragraphe 3. Vingt cycles charge-décharge ont été réalisés à vitesse de traverse constante $\left(5 \times 10^{-2} \mathrm{~mm} \cdot \mathrm{s}^{-1}\right)$, avec une contrainte maximale à chaque cycle de $30 \mathrm{MPa}$.

Les figures 3, 4 et 5 (noter que les échelles de couleur ne sont pas les mêmes) mettent en correspondance l'évolution du chargement mécanique (courbe blanche) et celui du profil des écarts de température observés sur l'axe médian de la face visible de l'éprouvette, respectivement pour les cycles 1 et 2, 9 et 10, 18 et 19. On constate un échauffement moyen croissant, perturbé par des fluctuations périodiques de faible

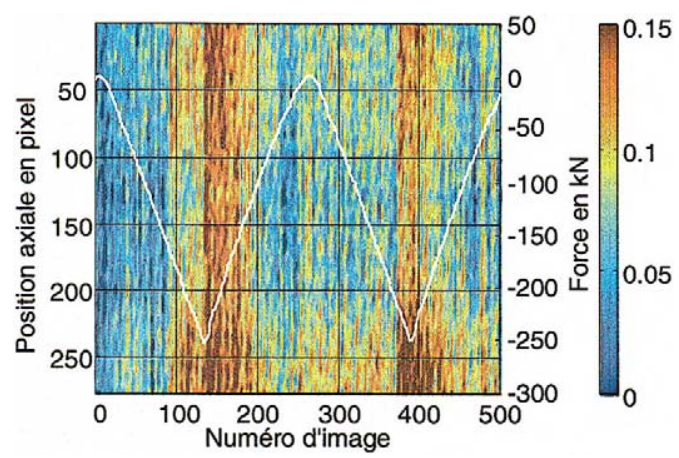

Figure 3. Evolution au cours du temps du profil de température de l'axe médian de l'éprouvette pour les deux premiers cycles.

Figure 3. Time evolution of the length-wise temperature profile for the two first cycles.

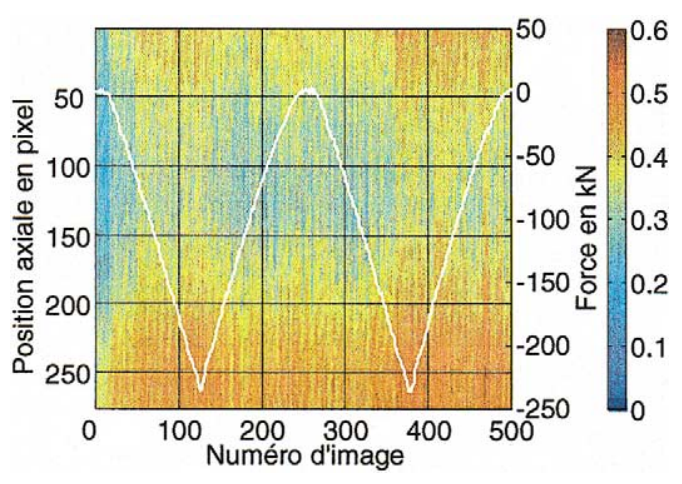

Figure 5. Évolution au cours du temps du profil de température de l'axe médian de l'éprouvette pour les cycles 18 et 19.

Figure 5. Time evolution of the length-wise temperature profile for the 18th and 19th cycles.

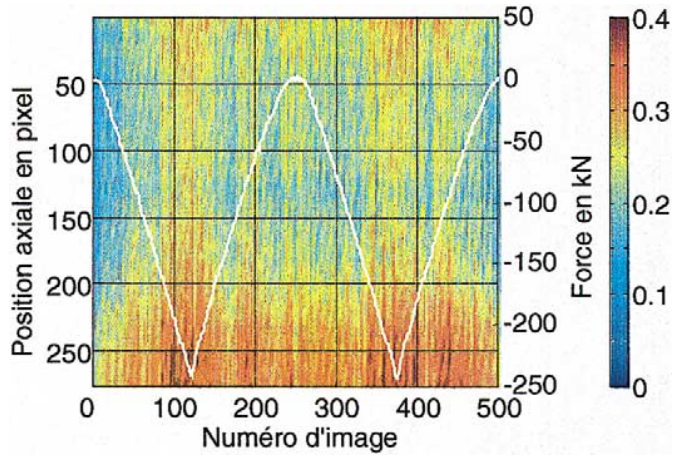

Figure 4. Evolution au cours du temps du profil de température de l'axe médian de l'éprouvette pour les cycles 9 et 10 .

Figure 4. Time evolution of the length-wise temperature profile for the 9 th and 10th cycles.

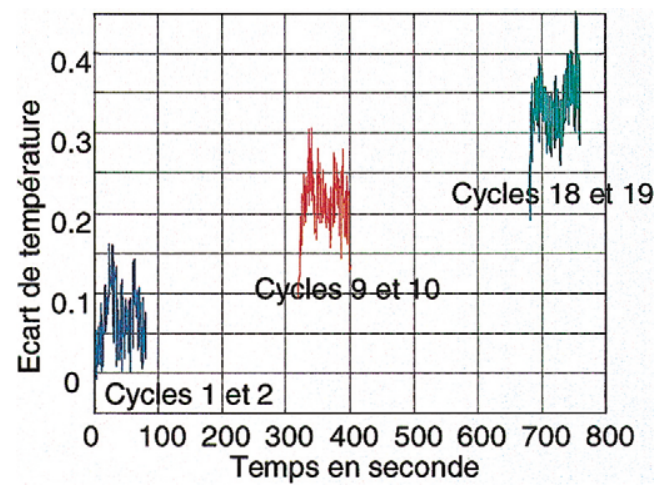

Figure 6. Evolution au cours du temps de la variation de température au point milieu de l'éprouvette.

Figure 6. Time evolution of temperature variation at middle sample point. 
amplitude en phase avec l'évolution de la charge. L'existence de cet échauffement peut légitimement être associée à un mécanisme dissipatif lié à l'état d'endommagement du béton. L'hypothèse de constance de l'endommagement, pendant les essais, s'est trouvée vérifiée au travers de deux essais successifs sur la même éprouvette : les résultats ont été identiques dans les deux cas.

Sur la figure 6, il apparait que l'élévation de température au point milieu de l'éprouvette dépasse $0,35^{\circ} \mathrm{C}$ au cycle 19 . On peut remarquer, également, une valeur de l'ordre de $0,07^{\circ} \mathrm{C}$ pour l'intensité maximale de la fluctuation périodique, en excluant les perturbations de mesure en début des cycles 1,9 et 18 . Ceci est en harmonie avec les prévisions théoriques des effets du couplage thermoélastique et avec les résultats mentionnés au paragraphe 4 .

\section{Conclusion}

Malgré la faiblesse des phénomènes thermiques mis en jeu, l'étude a permis d'identifier et de quantifier avec une relative précision les variations de température résultant de l'effet thermoélastique et les échauffements dus aux mécanismes dissipatifs liés à un état d'endommagement du béton obtenu par cycles gel-dégel. Pour les bétons endommagés et soumis à des chargements cycliques, il semble intéressant de tenir compte de ces phénomènes dans l'écriture des lois de comportement. En effet les faibles variations de température $\left(0,35^{\circ} \mathrm{C}\right.$ pour la dissipation) correspondent à la mise en jeu de puissance notables en raison de la forte capacité thermique massique des bétons. Elles peuvent être estimées avec les hypothèses de la section 5 à environ $10^{3} \mathrm{~W} \cdot \mathrm{m}^{-3}$. Un des objectifs ultérieurs des auteurs est de tester en ce sens la validité thermomécanique des lois de comportement proposées jusqu'ici pour les bétons endommagés et de les modifier si nécessaire.

\section{Références bibliographiques}

[1] Andrieux S., Bamberger Y., Marigo J.J., Un modèle de matériau microfissuré pour les bétons et les roches, J.Méca. Th. et Appl. 5 (3) (1986) 471-513.

[2] Ju J.W., On energy-based coupled elastoplastic damage theories: constitutive modeling and computational aspects, Int. J. Solids Structures 25 (7) (1989) 803-833.

[3] Mazars J., Berthaud Y., Une technique expérimentale appliquée au béton pour créer un endommagement diffus et mettre en évidence le caractère unilatéral, C. R. Acad. Sci. Paris, Série II 308 (1989) 579-584.

[4] Fremond M., Nedjar B., Endommagement et principe des puissances virtuelles, C. R. Acad. Sci. Paris, Série II 317 (1993) 857-864.

[5] Halm D., Dragon A., A model of anisotropic damage by mesocrack growth; Unilateral effect, Int. J. Damage Mech. 5 (1996) 384-402.

[6] Luong M.P., Vibrothermographie infrarouge d'un béton endommagé, C. R. Acad. Sci. Paris, Série II 301 (7) (1993) 459-464.

[7] Jacobsen S., Freeze/thaw cracking of $w / c=0,5$ prisms, Byggforsk report 9526, Norway (1999).

[8] Richard P. et al., Les Bétons de Poudres Réactives (BPR) à ultra haute résistance (200 à 800 MPa), Annales de l'ITBTP, Série : Béton 320 (532) (mars-avril 1995).

[9] Lemaître J., Chaboche J.L., Mécanique des matériaux solides, Dunod, Paris, 1985. 